\title{
TRIBUTACIÓN AMBIENTAL COMO INSTRUMENTO ECONÓMICO ANTE LA EXTERNALIDAD NEGATIVA PRODUCIDA POR LA CONTAMINACIÓN EN PERÚ
}

\section{ENVIRONMENTAL TAXATION AS AN ECONOMIC INSTRUMENT TO NEGATIVE EXTERNALITY PRODUCED BY POLLUTION IN PERU}

\author{
Paula Denisse García Malpica \\ Universidad Nacional Mayor de San Marcos \\ Investigadora Profesional egresada /Contadora Pública con mención en Gestión Tributaria de la Universidad Nacional Mayor de \\ San Marcos - UNMSM, Lima, Perú.
}

Correo del autor: paula.garcia.malpica@gmail.com

[Recibido: 06/12/2017 Aceptado: 20/04/2018]

\section{RESUMEN}

El objetivo de la investigación es describir cómo la tributación ambiental se puede aplicar como instrumento económico ante la contaminación, siendo esta una externalidad negativa. Sobre la base del objetivo en mención, se realizó un análisis descriptivo del fallo de mercado o market failure que representa la polución, y se presentó como herramienta de acción el instrumento económico tributario en materia ambiental, el cual actúa primero, como compensación por el daño realizado y segundo, como incentivo de comportamientos no perjudiciales para el medio ambiente. Del mismo modo, se presentaron las diversas cifras macros que representan el costo de degradación ambiental en el Perú en términos de Producto Bruto Interno, brindado por el Banco Mundial; se determinó cuáles son los problemas ambientales prioritarios que generan los costos económicos más significativos. De esta manera, se concluyó que los recursos naturales más afectados son el aire y el agua, que consecuentemente afectan a la salud y el bienestar general, y que la fiscalidad o tributación ambiental existe como instrumento económico para aminorar y evitar acciones dañinas que atenten a corto o largo plazo contra nosotros mismos y el medio en el que vivimos.

Palabras clave : Políticas ambientales, Impuestos, Cambio climático, Calentamiento global, Externalidades, Bienestar social, Desarrollo sostenible.

\begin{abstract}
The investigation allowed to describe how environmental taxation can be applied as an economic instrument in the face of pollution, this being a negative externality. A descriptive analysis of the market failure that represents pollution was carried out, and the tax economic instrument on environmental matters was presented as an action tool, which acts first as compensation for the damage performed and second, as an incentive for behaviors that are not harmful to the environment. Likewise, the various macro figures that represent the cost of environmental degradation in Peru in terms of Gross Domestic Product, provided by the World Bank, were presented. The main environmental problems that generate the most significant economic costs were determined. In this way, it was concluded that the most affected natural resources are air and water, which consequently affect the health and general well-being, and that taxation or environmental taxation exists as an economic instrument to reduce and avoid harmful actions that threaten ourselves in short or long terms and the environment where we live.
\end{abstract}

Keywords : Environmental policies, Taxes, Climate change, Global warming, Externalities, Social welfare, Sustainable development.

\section{INTRODUCCIÓN}

La contaminación es uno de los problemas más importantes, debido a que genera estragos irreversibles que afectan la salud ambiental, cuyo enfoque es integral, es decir, cómo impacta en los niveles de pobreza, seguridad alimentaria, nutrición, desarrollo sostenible y gestión ambiental. Sin embargo, aún no se ha llegado a un nivel óptimo en cuanto al uso eficiente de herramientas o instrumentos económicos que podrían aportar a la mejora de los aspectos mencionados, es decir la externalidad negativa ambiental que genera. ¿La tributación ambiental puede actuar como instrumento económico para hacerle frente a la contaminación?

Es así que, a través del método descrip- tivo, se detallará y analizará distintos documentos, estudios e informes relevantes emitidos por prestigiosas instituciones tales como el Comisión Económica para América Latina y el Caribe (CEPAL) de la ONU, Organización para la Cooperación y el Desarrollo Económicos (OCDE), la Organización de las Naciones Unidas (ONU) y el Banco Mundial (BM) para responder a la pregunta planteada.

C Los autores. Este artículo es publicado por la Revista Quipukamayoc, Universidad Nacional Mayor de San Marcos. Este es un artículo de acceso abierto, distribuido bajo los términos de la Licencia Creative Commons Atribución-NoComercial-Compartirlgual 4.0 Internacional.(http://creativecommons.org/licenses/by-nc-sa/4.0/), que permite el uso no comercial, distribución y reproducción en cualquier medio, siempre que la obra original sea debidamente citadas. 
Las externalidades ambientales son, como lo menciona Delacámara (2008) Una clase particular de externalidades (o efectos externos). No son más importantes que cualquier otro tipo de externalidad económica pero no sería posible encontrar argumentos racionales para justificar que lo son menos. Si se desea adoptar decisiones más complejas y, por lo tanto, mejores, las externalidades deberían ser debidamente cuantificadas e incorporadas en el marco de un análisis coste-beneficio de las decisiones públicas o privadas de la sociedad. En ese caso, pueden ser contabilizadas como costes de nuestras decisiones (aumento en la morbilidad como resultado de la contaminación atmosférica, daños en propiedades inmobiliarias en zonas rurales como resultado de inundaciones por una prevención inadecuada, etc., por citar algunos ejemplos) o, en su caso, como beneficios de las mismas (i.e., la reducción de la contaminación de las aguas en la cabecera de los ríos). (p. 5)

En efecto, contar con una base cuantificable sobre la cual se pueda sustentar el coste-beneficio de internalizar la contaminación ambiental, resulta primordial en cualquier tipo de planificación económica que se desee proponer. De esta manera al utilizar la acción de cuantificar tenemos datos precisos que evitan la subjetividad. Por ejemplo se puede criticar un autobús porque emite una gran cantidad de humo negro, pero la cuantificación permitirá precisar objetivamente qué tanta emisión de dióxido de carbono está generando en cierta cantidad de tiempo y cuánto daña al medioambiente. Cabe mencionar que no solo el ámbito de medición está presente, sino nace como segunda proposición el actuar ante tal situación para disminuir las cifras cuantificadas.
Se argumenta que el mayor obstáculo para incorporar estas externalidades, en el análisis económico de políticas públicas o decisiones privadas, tiene que ver con su dificultad para ser valoradas monetariamente. Se suele hablar de ellas en muchas ocasiones como efectos intangibles. Curiosa expresión si lo que se pretende sugerir es que una infraestructura física para el transporte o una unidad de energía obtenida a partir de la combustión de carbón son bienes tangibles y el ingreso hospitalario de ancianos con problemas asmáticos asociados al aumento de la concentración de contaminantes o el ruido asociado a la congestión urbana no lo son. Es posible que exista alguna razón para argumentar así, pero desde luego no es fácil de detectar. Nadie mejor que un responsable de las cuentas públicas sabe que las importaciones de productos derivados del petróleo suponen una importante salida de divisas, de modo similar a como la atención primaria en centros hospitalarios por dolencias asociadas a la contaminación atmosférica urbana consume igualmente un volumen no despreciable de recursos públicos. (Delacámara, 2008, p. 5)

El problema a tratarse es ¿Cómo hacerle frente a la contaminación desde el ámbito de las ciencias sociales-económicas, por medio de un instrumento económico efectivo que pueda internalizar los costos de tal externalidad y a su vez paliar el daño ambiental?

La hipótesis de mi trabajo se basa en que la tributación ambiental es un instrumento económico eficiente que permite internalizar los costos de las externalidad producidas por la contaminación y a su vez paliar el daño ambiental. Pues genera ingresos de finalidad extra-fiscal por el factor de 'contaminador-pagador' y modifica de esta manera el comportamiento de los consumidores, además de, en el caso de los incentivos tributarios, promover el uso o creación de nuevas tecnologías 'verdes'.

Se realizó una investigación descriptiva, teniendo como unidad de análisis la situación de desarrollo sostenible del Perú en general, es decir qué avances y cómo se encuentra el país en términos ambientales reflejado en las investigaciones realizadas en el tema, los niveles de contaminación afectados prioritariamente, qué recursos son los han sido más afectados, el PBI en cuanto al desarrollo económico por el cual se mide el bienestar del país y los costos de degradación ambiental que son las consecuencias directas del daño ambiental.

La investigación es tipo no experimental ya que no se ha intervenido directamente con ninguno de los datos presentados en el presente artículo.

Por otro lado, la limitación de esta investigación es que no se cuenta con datos macro actualizados sobre los costos de degradación ambiental en porcentaje del PBI, en salud y calidad de vida con respecto a otros países y costos de daño a la salud ambiental en soles, ya que esta información solo ha sido analizada de esta manera en el 2007 por el Banco Mundial, en el estudio denominado Análisis Ambiental del Perú: Retos para un Desarrollo Sostenible realizado por la Unidad de Desarrollo Sostenible Región de América Latina y el Caribe.

Los resultados se exponen en los siguientes puntos:

\section{Externalidad negativa y justificación económica}

Traduciendo la contaminación ambiental a términos económicos, se le considera como una 'falla de merca- 
do' o 'market failure' que genera diversas externalidad negativas. Como ejemplo práctico, una empresa que fabrica zapatos, utiliza una maquinaria que genera polvo que ensucia más de lo normal el piso de unas oficinas de la empresa vecina, por lo cual esta segunda empresa tendrá que asumir un coste adicional por limpieza que está fuera de su propio control. Pero no solo ello, sino que residuos de esta máquina pueden estar produciendo daños colaterales en la salud de los trabajadores de la segunda empresa, por lo cual tendrá que cubrir los gastos médicos extraordinarios que se generen de ellos y por lo tanto aumentar su presupuesto designado para este fin. Más aún, los químicos que emite la máquina causan un daño al medioambiente. De esta forma se demuestra las diversas consecuencias o externalidades negativas que trae la contaminación.

Es así, que la contaminación ambiental es, de acuerdo con Bator (1998)

Un fallo de mercado que produce costes sociales, debido a que el bien ambiental es un bien público y dichos costes, también llamados por los economistas externalidades negativas, son costes sociales, ya que afectan a la sociedad en su conjunto como sujeto titular de los derechos de disfrute del bien ambiental. Básicamente este tema ha sido objeto de estudio de la economía debido a que las externalidades negativas se consideran un fallo del mercado y la contaminación sin duda es el tipo más resaltante de las externalidades negativas. (pp. 73-74)

$\mathrm{Y}$ es por ello que resulta importante analizar cómo los efectos de la contaminación inciden en la economía y el bienestar social. Además de conocer y aplicar los instrumentos más adecua- dos para las diferentes situaciones que se puedan presentar.

Instrumento económico tributario

La tributación ambiental es un instrumento económico importante para dos fines principalmente:

- El primer fin, compensación por el daño ambiental es decir el principio de contaminador-pagador; y

- el segundo, como incentivo de comportamientos no perjudiciales que contribuyan positivamente al medioambiente.

Por lo cual, tenemos este instrumento económico tributario que puede ser aplicado, con fin ambiental, para hacerle frente a la contaminación. Para realizarlo de manera efectiva, es prioritario conocer cuáles son los problemas ambientales que aquejan a nuestro país y cómo están afectando desde hace años a la economía nacional. De esta manera podemos tomar medidas de acción para paliar el daño ambiental que es causado a diario y que tanto en el corto como largo plazo traerá consecuencias graves para los ámbitos sociales, económicos y de salud de la sociedad.

La protección ambiental necesita instrumentos de intervención de índole administrativo, penal y, desde luego, tributario. La tributación se centra con fuerza por su cualidad preventiva a diferencia de las otras formas de intervención. La tributación es uno de los instrumentos económicos que existen y que posibilita la internalización de costos producido por la contaminación. Por eso, para saber su ubicación dentro de todos los instrumentos económicos más conocidos, se tomó los estudios realizados por Alberto Gago y Xavier Labandeira. Dichos profesores han precisado que la OCDE (1989) define a estos instrumentos de política, como aquellos que producen modificaciones en el comportamiento ambiental de los agentes, mediante el simple juego de los incentivos financieros y del mercado. (Yacolca, 2012, p. 65)

Desarrollo y costos de la degradación ambiental

La palabra desarrollo se ha usado en el Perú desde hace ya varios años, sin embargo, gran parte de la población no suele percibir esa mejora progresiva que implica en casi ningún sentido, por lo contrario, se ha tergiversado su conceptualización hacia un bienestar solo de algunos sectores sociales quienes son los más beneficiados con tal 'desarrollo', que rápidamente se asocia únicamente con el ámbito económico.

Cabe destacar que el medioambiente prácticamente está considerado poco relacionado al término expuesto. A pesar de la gran cantidad de recursos por proteger y las consecuencias ya tangibles como las inundaciones y grandes pérdidas por inundaciones, llamados huaycos, que hay todos los años, sin llegar al tema de fondo, la causa de aquellos eventos. El desarrollo económico del Perú, según CEPAL/OCDE (2017, p.79) "se ve cada vez más afectado por los costos asociados al impacto de eventos naturales extremos y del cambio climático, que se suman a las consecuencias económicas de la degradación ambiental. Los eventos extremos, ejemplificados por los episodios de los fenómenos de El Niño (1982-1983) y de La Niña (1997-1998), tuvieron efectos catastróficos tanto en términos de pérdida de vidas humanas como de damnificados; también tuvieron repercusiones económicas en los sectores pesquero y agrícola y en la infraestructura, lo que sitúa al Perú entre los 
países más expuestos a los riesgos ambientales (Bello, Ortiz y Samaniego, 2014). Según diversas estimaciones, el cambio climático provocaría una reducción de 4,3 puntos porcentuales del PIB -Producto Bruto Interno, también denominado PBI- hacia 2025 (CAN, 2008) y de 6 puntos hacia 2050 (CAN, 2008). El Banco Interamericano de Desarrollo y la CEPAL (BID/ CEPAL, 2014) proyectan pérdidas del PIB de alrededor del 15\% en el presente siglo." Es así que se evidencia las consecuencias que genera el daño ambiental tanto en el ámbito económico y como en salud; y Perú es uno de los más riesgosos. Asimismo, no se debe dejar de lado el propósito de protección de la biodiversidad que posee nuestro país en relación con nuestros recursos.

La sostenibilidad es una filosofía necesaria por aplicar en nuestro país, puesto que en el mundo globalizado en el que vivimos, las Naciones Unidas por ejemplo ha propuesto los ODS - Objetivos de Desarrollo Sostenible. Se hace necesario usar nuestro grandes recursos naturales de manera prudente y sostenible, teniendo en cuenta que muchos de ellos no son renovables, aprovechar la bonanza económica que nos pueden dar pero sin abusar, además de contribuir a la reducción del daño ambiental, según el mismo informe CEPAL/OCDE (2014, p. 80 ) esta necesidad "ha ido adquiriendo cada vez más relevancia en el Perú. No obstante, desde el comienzo del desarrollo de la normativa ambiental se han producido contradicciones fruto de la percepción de que esta podría obstaculizar la actividad económica. Esto condujo a la promulgación de la Ley Marco para el Crecimiento de la Inversión Privada (Decreto Legislativo 757) y la Ley de Promoción de Inversiones en el Sector Minero (Decreto Legislativo 708), de conformidad con las cuales se modificó el Código de Medio Ambiente y de los Recursos Naturales de 1990 y se dejaron sin efecto varias de sus disposiciones. Con el objeto de eliminar las trabas a las actividades económicas, en virtud de la primera de estas se formalizó la sectorialización administrativa de la gestión ambiental. Desde la creación del Consejo Nacional del Ambiente (CONAM) en 1994 y, en especial, del Ministerio del Ambiente en 2008, se ha reforzado la política ambiental con el propósito de establecer una relación más coherente entre la protección del medio ambiente y la actividad económica. Con el mismo objetivo, se establecieron el sistema de evaluación del impacto ambiental y el sistema de ventanilla única para proyectos de inversión de gran envergadura, que funcionan en el marco del Servicio Nacional de Certificación Ambiental para las Inversiones Sostenibles (SENACE) — creado en 2012 y adscrito al MINAM - organismo que asumirá progresivamente las funciones de las autoridades sectoriales a partir de 2015. En 2012 se creó la Comisión Multisectorial, facultada para elaborar propuestas de leyes y políticas orientadas a mejorar las condiciones ambientales y sociales en las que se desarrollan las actividades económicas, especialmente de las industrias extractivas (Resolución Suprema 1892012-PCM) (...)."

De esta manera, desde el 2008, con la creación del Ministerio del Ambiente, la institucionalidad en materia medioambiental se ha ordenado y adquirido fuerza, logrando propuestas de ley y resoluciones a favor del desarrollo económico sostenible. Tales medidas, con el paso del tiempo están tomando mayor protagonismo, lo cual se puede ver claramente reflejado en los incentivos que se están dando para desarrollo e investigación de tec- nologías limpias actualmente. Sostenibilidad y desarrollo sostenible

A raíz de evaluaciones realizadas a nivel mundial, Bybee (1991) catalogó la situación como una "emergencia planetaria”, lo cual pone en peligro inminente a todas las personas en el mundo. De esta manera nace el concepto de sostenibilidad.

El informe Brundtland que apoya la postura de sostenibilidad o sustentabilidad a la par y en favor del desarrollo económico a nivel global, en el cual prime la armonía con el medioambiente, es decir la renovación de un recursos natural no debe exceder los límites de su propia explotación, fue realizado para la Organización de las Naciones Unidas (ONU) por distintos países en 1987. Específicamente, de acuerdo a la Comisión Mundial del Medio Ambiente y del Desarrollo (CMMAD, 1988), el concepto es el siguiente: "El desarrollo sostenible es el desarrollo que satisface las necesidades de la generación presente sin comprometer la capacidad de las generaciones futuras para satisfacer sus propias necesidades".

\section{Análisis socioeconómico}

La economía en el mundo actual es la base que sostiene a la sociedad, en sus diversas expresiones, ya sea con fin social o lucrativo, es por ello que se le hace referencia como uno de los principales enfoques de investigación para sustentar los fines planteados. Para realizar un análisis socioeconómico a nivel macro, se presenta un estudio realizado en el Análisis Ambiental del Perú (AAP), elaborado por el Banco Mundial (2007), entidad global que brinda entre, diversas funciones y objetivos, estadísticas sobre diversos temas, tales como el medioambiente y economía, "para identificar 
los problemas ambientales que están asociados con los costos económicos más significativos (Larsen y Strukova). Tal trabajo estimó que el costo económico de la degradación ambiental, reducción de los recursos naturales, desastres naturales, servicios ambientales inadecuados (tales como sanidad inadecuada) sumaban 8,2 billones de soles, equivalentes al 3,9 por ciento del producto bruto interno (PBI) en 2003. El análisis muestra que los problemas asociados con la degradación ambiental de mayor costo son, en orden decreciente: el inadecuado abastecimiento de agua; la sanidad e higiene; la contaminación atmosférica urbana; los desastres naturales; la contaminación por plomo; contaminación de aire en locales cerrados y la degradación del suelo agrícola. Los costos de la deforestación y la inadecuada recolección de desechos sólidos en los hogares son sustantivamente menores que las categorías anteriores. Es importante notar que estos estimados están basados en suposiciones muy conservadoras que apuntan a reducir las dudas relacionadas con datos restringidos." Lo afirmado anteriormente se muestra en la Figura 1.

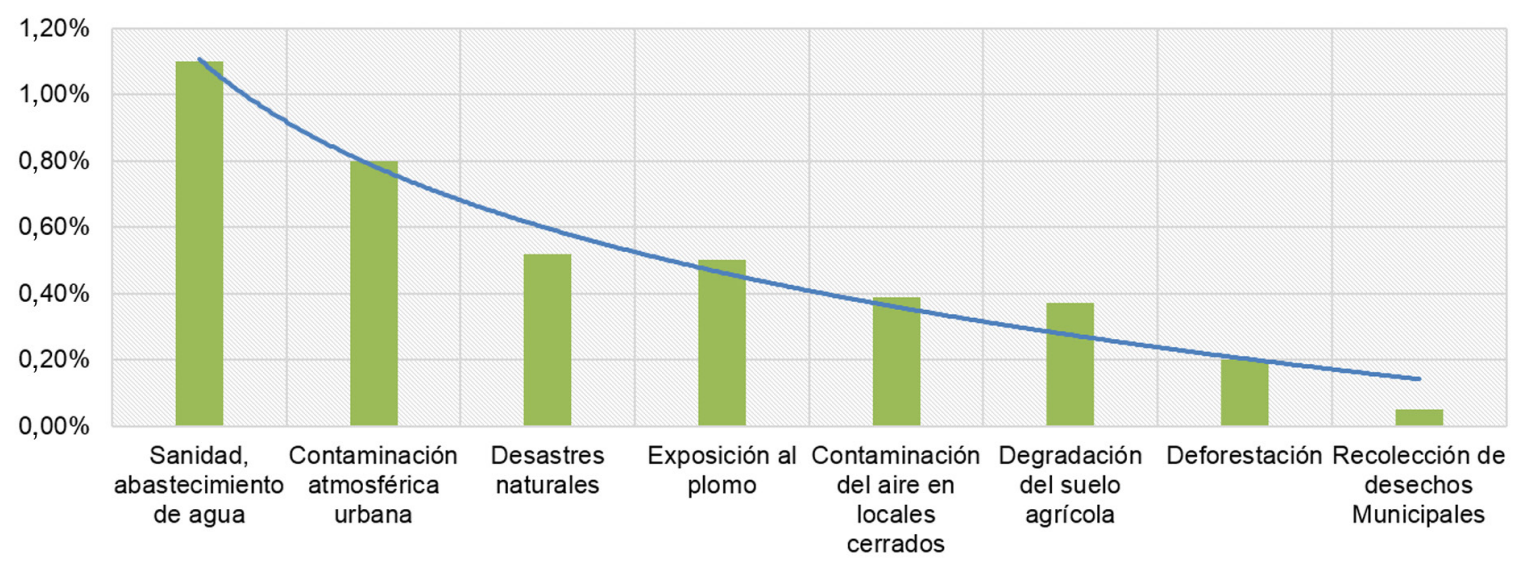

Figura 1. Costos de Degradación Ambiental Anual (\% del PBI).

Fuente: Larsen y Strukova (2005), citado por Banco Mundial (2007) - Elaboración propia.

La medición del daño causado al ambiente se transforma en una necesidad perenne para plantear medidas correctas, de acuerdo a Pazos (2004), "la degradación ambiental se produce debido al aumento del consumismo, que a la fecha actual se ha tornado realmente incontrolable, y a través de la denominación de 'ciclo superproducción-consumo-eliminación de desechos'. Es así que genera que nuestro mundo presente progresivamente, una escasez de recursos renovables para sostener al ambiente $y$ al género humano. Se presenta la afinidad de tener constante crecimiento económico sin importar los costos ecológicos en medio de la globalización dentro de la economía liberal; así, los recursos naturales se agotan cada vez más conduciendo a diversas falencias que afectan a nuestro propio ambiente." Es así, que la medición de los costos ambientales se puede lograr de acuerdo al impacto que genere en el crecimiento económico en cierto plazo. Entonces, se plantea el modelo de relacionar las variables económicas como por ejemplo PBI con Costo ambiental de cierto tipo y en determinadas zonas de acuerdo al impacto que el elemento ambiental esté generando en el ámbito enlazado que haya sido previamente observado y probado. De esta manera, llegamos a la crítica situación medioambiental actual que cada vez se va haciendo más visible a través de sus efectos. El aire que se res- pira ahora mismo, por ejemplo, en la ciudad de Lima, no es el mismo que el de hace unos 10 años, crecer de manera desordenada sin tomar en cuenta la sostenibilidad de cada acción que realizamos, nos trae como consecuencia la degradación ambiental que soporta nuestro país. En efecto, "el costo de la degradación ambiental en el Perú es más alto que en otros países con niveles de ingreso similares. Estudios del costo de la degradación ambiental llevados a cabo en Colombia, un país de América Latina con nivel de ingreso medio-alto, y en otros países de África del Norte y el Medio Oriente con niveles de ingreso bajo-medio, demuestran que el valor monetario de la elevada morbilidad y mortalidad 
típicamente se encuentra debajo del $2 \%$ del PBI en estos países, cuando en Perú es del 2.8\% del PBI ." (Banco Mundial 2007, p. 13)

Con lo mencionado anteriormente, se puede deducir que el Producto
Bruto Interno (PBI) no es distribuido de igual manera en cuanto a los fines ambientales a los que se destina, en comparación a países de posición y situación económica similar, ya que ellos poseen sus costos de degradación ambiental en menor porcentaje que nuestro país tal como se muestra en la Figura 2. Además, que no se hacen los esfuerzos debidos para paliar los daños causados por el calentamiento global y el efecto invernadero, producto de la contaminación.

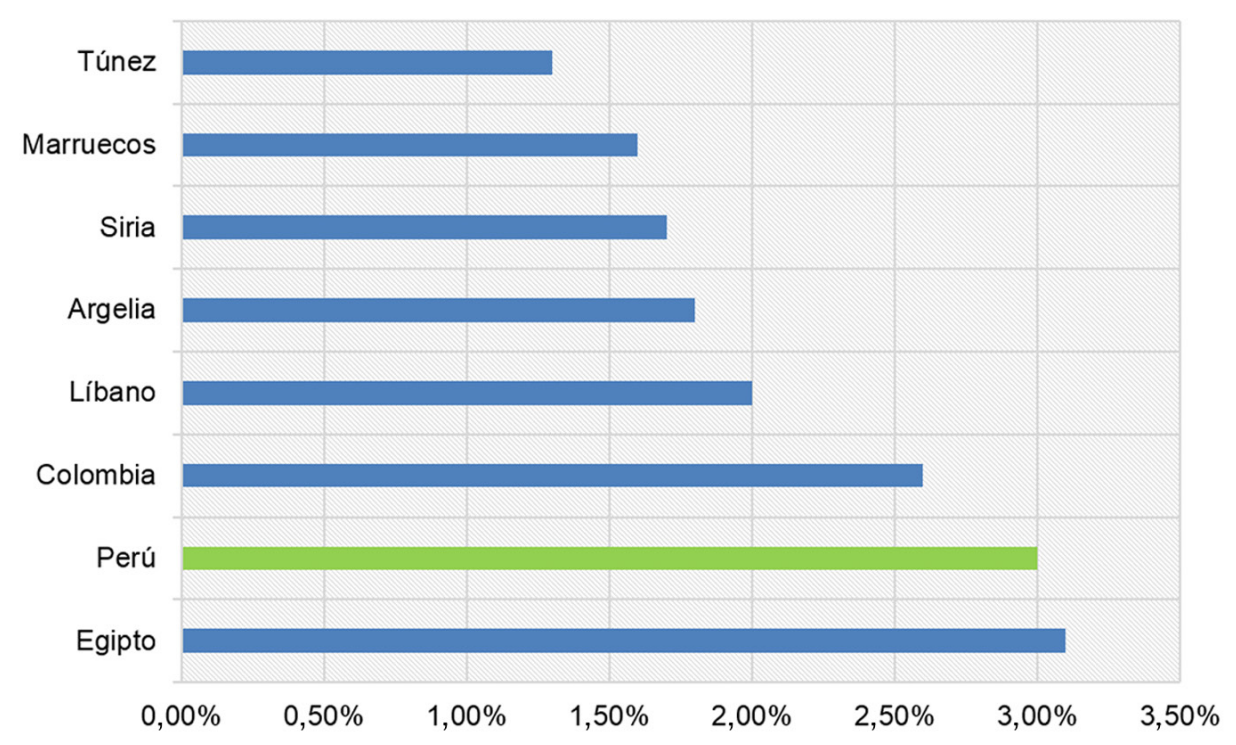

Figura 2. Costos de Degradación Ambiental Anual Costos de Degradación Ambiental (Salud y Calidad de Vida) (\% del PBI).

Fuente: Banco Mundial (2007) - Elaboración propia.

"La carga de estos costos recae más duramente en los grupos vulnerables. Las poblaciones vulnerables son expuestas a los más altos riesgos ambientales que los grupos con más altos ingresos y carecen de los recursos para mitigar esos riesgos. Es crucial resaltar la importancia de la degradación ambiental como una barrera para reducir la desigualdad." (Banco Mundial 2007, p. 13)

Esencialmente, sigue sucediendo. Un ejemplo claro acerca de ello, como se mencionó en párrafos anteriores, lo podemos observar todos los años, con los afectados tanto en salud como económicamente por los huaycos, que bien sabemos se puede aplicar un plan de prevención a través de infraestructura adecuada y desarrollo urbano, teniendo en cuenta también que la intensidad de los huaycos se deriva de la cantidad de lluvias que haya y estas se originan por la evaporación del agua del mar y lagunas debido al aumento descontrolado de la temperatura, producto del calentamiento global.

Los impactos negativos en la salud según el BM (2007) “representan más del 70 por ciento de los costos de la degradación ambiental. Aumentos en la morbilidad y la mortalidad resultantes de la contaminación atmosférica urbana y la exposición al plomo, inadecuado abastecimiento de agua, sanidad e higiene; y contaminación del aire en locales cerrados tienen un costo estimado de 5,85 billones de soles" (p.15). La salud es el factor que se ve más afectado por las consecuencias expuestas en la Figura 3. Y se recalca nuevamente que estos efectos se aplican con mayor intensidad a las poblaciones de bajos recursos económicos quienes se encuentran, por lo tanto, en situación de mayor vulnerabilidad. 


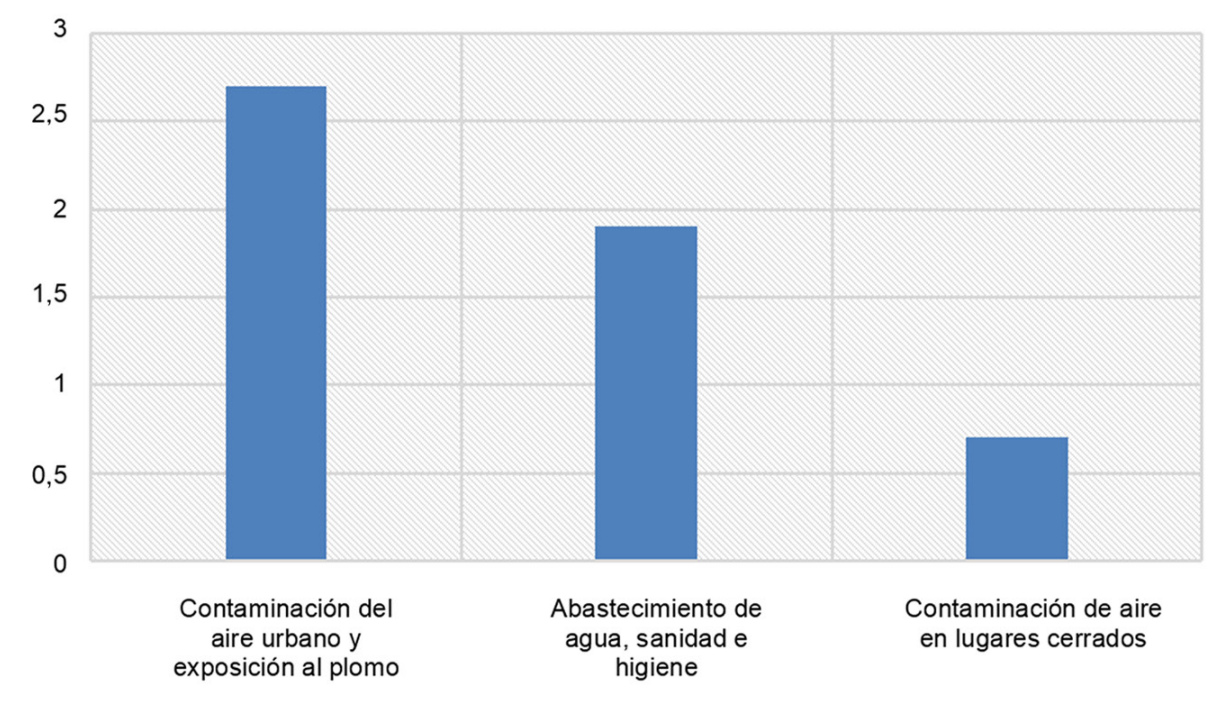

Figura 3. Costos del Daño a la Salud Ambiental en el Perú (miles de millones de soles por años). Fuente: Banco Mundial (2007) - Elaboración propia.

Esencialmente, sigue sucediendo. Un En efecto, los costos del daño a la salud ambiental resultan ser en orden de prioridad para la contaminación del aire urbano y exposición al plomo, situación que podemos comprobar a diario al estar en las grandes avenidas congestionadas, por ejemplo. Seguidamente tenemos el abastecimiento de agua, sanidad e higiene; en aún varias provincias de Perú el acceso al agua es casi exclusivo, para poder llenar unos baldes de agua, las personas deben caminar kilómetros. Según un artículo publicado en el Día Mundial del Agua (Pimentel, Palacios, 2017), "aún antes del impacto del Fenómeno El Niño Costero en Perú desde inicios de 2017, ocho millones de peruanos carecían de los servicios de agua potable y alcantarillado. Por ejemplo, en la provincia de Utcubamba (región Amazonas), 27 mil de los 59 mil habitantes que viven en la ciudad solo cuentan con agua potable dos horas cada semana. Además, se menciona que un informe de 2002 de la Organización Panamericana de la Salud señala que el $10 \%$ más pobre de los centros urbanos del país pagaba el doble que el $10 \%$ más rico por el uso del agua. Otro estudio, publicado por el Programa de las Naciones Unidas para el Desarrollo (PNUD), dice que el Perú es el país latinoamericano con mayores niveles de desigualdad en cuanto al acceso al agua potable en el hogar."

Evaluando en cifras la situación presentada en el párrafo precedente, "los más altos costos de la degradación ambiental en el Perú, son en orden decreciente: inadecuado abastecimiento de agua; sanidad e higiene; contaminación atmosférica urbana; desastres naturales; exposición al plomo; contaminación del aire en locales cerrados; degradación de la tierra; deforestación e inadecuada recolección municipal de desechos. Combinados, estos problemas ambientales cuestan 8,2 billones de soles, o 3,9 \% del PBI. Las poblaciones más vulnerables cargan desproporcionadamente el peso de este costo." (BM, 2007, p. 33)

Sobre la salud ambiental, que como se observó está claramente afectada en el Perú y necesita de manera urgente atención sobre todo en las zonas rurales quienes son los más perjudicados, la Organización Mundial de la Salud (OMS, s.f.) afirma que "está relacionada con los factores físicos, químicos y biológicos externos de una persona. Es decir, que engloba factores ambientales (...)", los cuales podrían afectar a la salud. Además, menciona que está basado en principalmente (i) prevenir cualquier tipo de enfermedades y (ii) crear ambientes apropiados para desarrollar un nivel de salud óptimo. Concluye que dentro de este concepto, están excluidos los que no tengan relación con el medioambiente y los que estén relacionados con economía, ámbito social o la genética.

Sobre el saneamiento ambiental, podemos citar lo que comenta Prindle (1966, p.337), "en el mundo actual, la salubridad del ambiente es un objetivo social básico, un concepto positivo cuya finalidad no se limita a salvaguardar la salud sino también a promover el valor íntegro de la vida comunal." El ambiente que nos rodea 
como personas, lo hace de igual manera para cada ser vivo, por lo cual se convierte en un bien social que va a determinar el bienestar común de la sociedad global. De ahí deriva su importancia.

\section{Situación actual}

En el período que se analiza en el estudio Evaluaciones del desempeño ambiental: Perú por la CEPAL/OCDE (2017), desde los inicios del 2000 hasta 17 años posteriores, "se han logrado avances notables en esa dirección: se han fortalecido tanto el marco legal como la institucionalidad ambiental, se ha mejorado el sistema de información, se han aplicado herramientas de gestión que integran aspectos económicos y ambientales y se ha avanzado en una estrategia de crecimiento verde. Los esfuerzos en materia de educación sobre el medio ambiente, fomento de la participación ciudadana y acceso a la justicia ambiental también están contribuyendo a crear una sociedad más consciente y activa en defensa del desarrollo sostenible." (p.3) De esta manera, se hace evidente que los ámbitos de la legalidad, educación, participación ciudadana e institucionalidad ambiental han mejorado de manera considerable. Logrando una difusión y aceptación adecuadas, creando personas más alertas y con propuestas para la desarrollar la sostenibilidad en el Perú. Una de las grandes metas en las cuales se ha avanzado de manera progresiva y en la cual se sigue incidiendo en mayor medida actualmente.

Adicionalmente, menciona la CEPAL/OCDE (2017), "el Perú ha crecido a una tasa promedio superior al $6 \%$, sostenida en la estabilidad macroeconómica, la apertura comercial y el influjo de inversión extranjera fuertemente atraída hacia la explo- tación de los recursos naturales. En paralelo a la tutela de las condiciones necesarias para la inversión privada en distintos sectores productivos, se ha fortalecido el marco institucional ambiental, así como la legislación en materia de gestión ambiental y conservación de los recursos naturales, aunque no se ha logrado desvincular los procesos de degradación ambiental ni sus costos asociados del crecimiento económico." (p.74) A pesar de los esfuerzos y logros mostrados, estas acciones no han incidido eficiente ni efectivamente en los costos de la degradación ambiental que afectan de manera creciente a la economía del país. Es decir la creciente fortaleza de las entidades y del marco legislativo relacionada al medioambiente deben apuntar a disminuir cuantitativamente el daño ambiental y los costos que él genera, sino podría ser considerada como algunas normas que tal vez están muy bien escritas, estructuradas $\mathrm{y}$ aprobadas pero que realmente no se cumplen o no logran de manera tangible los efectos deseados.

Continuando con las estimaciones brindadas por el CEPAL en conjunto con la OCDE, "a inicios del período de análisis los costos de los daños ambientales ascendían a un 4\% del PIB, el $70 \%$ de ellos asociados a efectos sobre la salud por enfermedades transmitidas por el agua, contaminación atmosférica en ciudades, exposición al plomo y contaminación intradomiciliaria. Hay también estimaciones de la contaminación ambiental minera sobre los recursos hídricos que varían entre el $0,4 \%$ y el 0,7\% del PIB a mediados de la década de 2000. Aunque la información es muy parcial, existen indicios de reducción de algunos costos ambientales con posterioridad a 2006, particularmente los asociados a la contaminación atmosférica. De otro lado, los montos requeridos para la remediación de pasivos mineros, que casi alcanzaban el 2\% del PIB a inicios del período de análisis, han ido creciendo en paralelo al número de sitios contaminados identificados por el Ministerio de Energía y Minas (MINEM), que superaban los 8600 en 2015." (CEPAL/OCDE, 2017, p.74)

Por consiguiente, se hace materia de estudio la tributación como medio para combatir esas externalidades negativas que genera la contaminación, ya que se le considera como un instrumento económico ante esta situación que genera costos con efectos en el PBI. Más aún cuando se cerciora a través de las afirmaciones expuestas por el CEPAL en conjunto con la OCDE, que Perú es el primer país de Sudamérica en tener mayores costos relacionados a los desastres naturales, por lo que se comprueba que es un tema que debe ser agendado prioritariamente. En consecuencia, "consciente de la dependencia de su economía en los recursos naturales y del efecto de los costos ambientales sobre el bienestar, el Perú ha sentado las bases para su adhesión a la Declaración de Crecimiento Verde de la OCDE, mediante la discusión de una estrategia nacional de crecimiento verde y la adopción de contribuciones previstas determinadas a nivel nacional (CPDN) en materia de reducción de emisiones de gases de efecto invernadero, entre otras cosas. No obstante, aún persiste la falta de coherencia entre diversas políticas, planes y estrategias de desarrollo y las metas ambientales, las fallas de coordinación horizontal o vertical entre las distintas instituciones del Estado y la dificultad de compensar las medidas de promoción de las inversiones con la eficiencia y eficacia de las políticas ambientales." (CEPAL/OCDE, ídem)

En efecto, se suele considerar como 
una de las mayores falencias que dificulta la promulgación de leyes o efectividad de las mismas es el ordenamiento y los diversos intereses del Estado, que pueden ralentizar este tipo de procedimientos legales. $\mathrm{O}$ tal vez, por ejemplo, al establecer una norma de carácter tributario, no se tiene en cuenta todas las posibles consecuencias económicas que pueden causar grandes estragos a largo plazo.

Por otro lado, las metas ambientales no están del todo alineadas teniendo en cuenta, ejemplificando, a los ODS - Objetivos de Desarrollo Sostenible establecidos por la ONU que tienen como prioridad promover a la colaboración y el bienestar de la población global a través de los lineamientos trazados, muchos de ellos en la actualidad son desconocidos para los propios funcionarios estatales.

\section{Impuestos ambientales en Perú}

"Los ingresos tributarios del Perú aumentaron del $14,5 \%$ del PIB en 2000 al $18,3 \%$ en 2013 (OCDE/CEPAL/ CIAT/BID, 2015). No obstante, son relativamente bajos en comparación con los ingresos de esta índole de los países miembros de la OCDE y de sus pares de América Latina y el Caribe, cuyos promedios ascendían al 34,1\% y al 21,3\% del PIB, respectivamente, en 2013. En ese contexto, los impuestos ambientales permitirían incrementar la recaudación y ofrecer incentivos para la aplicación de una estrategia de crecimiento verde, conforme a los principios de quien contamina o usa, paga." (CEPAL/OCDE, 2017, p. 84) Efectivamente, entre los objetivos y beneficios de la aplicación de impuestos ambientales se tendría cierto aumento en la recaudación hasta que el comportamiento del contribuyente se vaya ajustando de acuerdo a como se apliquen los lineamientos que se planteen y sobre todo la paliación del daño ambiental causado por los agentes contaminantes a quienes se les impondría el principio de contaminador-pagador por el efecto dañino que esté causando a nuestro ecosistema, estos ingresos deberán ser destinados a fines exclusivamente ambientales, apuntando hacia un desarrollo sostenible sin abusos, explotación inconsistente de recursos naturales ni atropello de derechos.
"Sin embargo, el sistema tributario actual no acompaña una estrategia de crecimiento verde y en 2012 los ingresos procedentes de impuestos relacionados con el medio ambiente apenas representaban el 0,6\% del PIB, porcentaje muy inferior a los promedios de los países miembros de la OCDE e incluso de otros países de la región. Además, en el período analizado no se observa un incremento (...); todos estos tributos son impuestos sobre los combustibles y las gasolinas, aunque algunos también se aplican a los automóviles." (CEPAL/OCDE, ídem) Como se puede observar (Figura 4), Perú está casi al último de todos los países en comparación, analizando los ingresos que se obtiene debido a los impuestos que se aplican relacionados con el medioambiente. En caso concreto, el único tipo de impuesto relacionado fue establecido para el sector energía y la recaudación es menor al $1 \%$ del PBI. Además, está incluido dentro del impuesto selectivo al consumo.

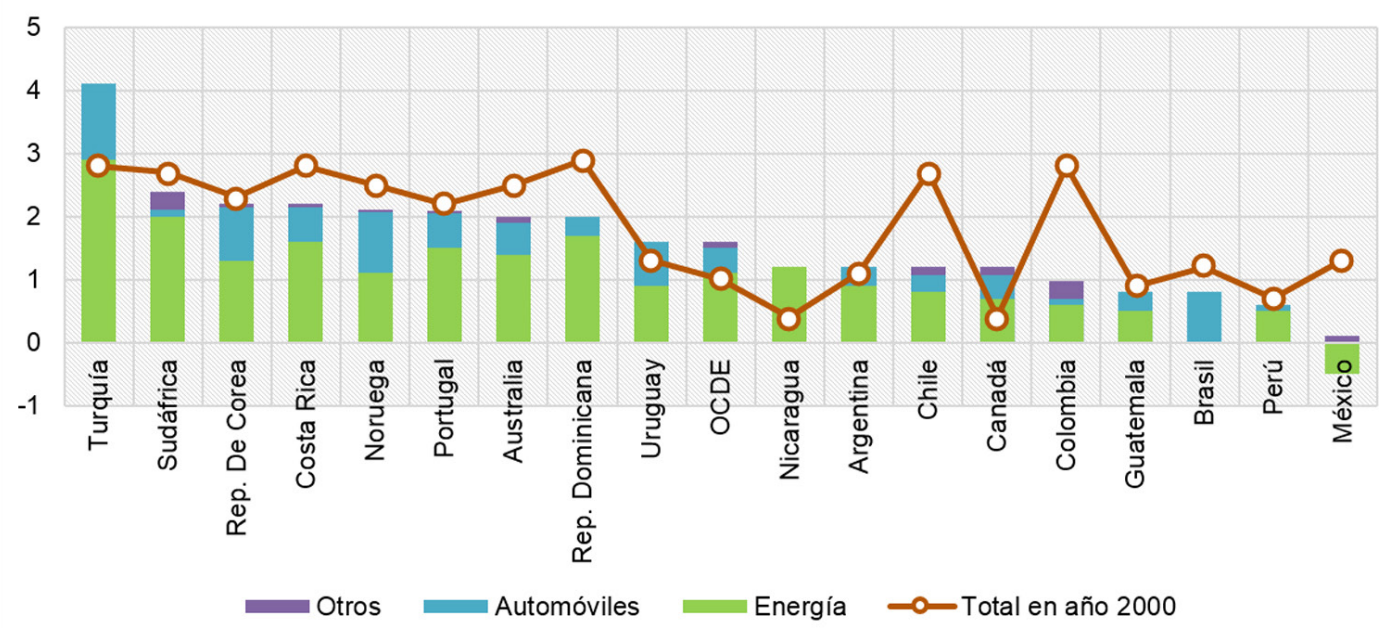

Figura 4. Ingresos procedentes de impuestos relacionados con el medio ambiente, 2000-2013 (\% del PBI).

Valores 'Total' correspondientes al año 2000, y para Perú, 2012.

Fuente: CEPAL/OCDE (2017) - Elaboración propia. 
"El impuesto selectivo al consumo (ISC) es un impuesto específico que grava la venta en el país, a nivel del productor y del importador, de la gasolina para motores, el keroseno y los carburo-reactores tipo keroseno utilizado en reactores y turbinas, los gasóleos y la hulla. Constituye un ingreso directo para el gobierno nacional. En 2013-2014, este tributo, que es el más importante relacionado con el medio ambiente, registró una disminución real y como proporción de los ingresos tributarios, debido a la reducción del gravamen aplicable a las gasolinas y los gasóleos (Decreto Supremo 316-2014-EF)." (CEPAL/ OCDE, 2017, p. 85) En el 2016, el ISC aplicado para los gasoholes se incrementó en 32\%, para el B2, 25\% y para B5, aumentó 9\%, medida promulgada por el Decreto Supremo No 306-2016-EF, calificada por el propio Ministro de Economía de esa fecha, Alfredo Thorne, como un aporte positivo con objetivo verde, a través de la siguiente frase: "Hay un compromiso medioambiental por lo cual como Ejecutivo le damos beneficios a las gasolinas y los diésel que hoy tienen menor contenido de azufre versus los que tienen mayor contenido" ("MEF elevó el ISC", 2016) Por ello, se rescata este tipo de medidas que contribuyen con el fin de protección ambiental a través de la modificación o ajuste de comportamientos del consumidor o usuario final del objeto contaminante. Cabe resaltar también los incentivos actuales que se dan para las empresas que realicen o estén involucradas en proyectos o iniciativas que impliquen investigación o uso de tecnologías eco-friendly. De esta manera, apuntamos como sociedad a ser un país que respete su propio ecosistema y recursos naturales, y practicar la sostenibilidad efectivamente.

\section{CONCLUSIONES}

Los instrumentos económicos ayudan a resolver o amortiguar las fallas de mercado que se producen inevitablemente. Como se ha presentado en el apartado anterior, la contaminación es el mejor ejemplo de externalidad negativa y también es uno de los problemas más importantes a resolver, ya que daña la salud ambiental y no solo ello, sino que genera costos que afectan a todos como economía nacional y global.

A pesar de las recomendaciones y cifras mostradas tanto por el BID, CEPAL, OCDE o el Banco Mundial, no se han tomado medidas en términos de políticas públicas para accionar en temas ambientales.

El market failure, junto con sus efectos negativos, puede ser aminorado a través de la herramienta de la tributación ambiental. Que como mencionó Bator, la contaminación está considerada como el mejor ejemplo de externalidad negativa. Por lo tanto, un sistema tributario que comprenda ámbitos ambientales bien estructurados y objetivos claros, puede actuar como instrumento económico para hacerle frente a la contaminación.

Se evidencia con lo que menciona Yacolca, sobre la importancia de contar con instrumentos de todo índole que tengan como objetivo la protección ambiental, tanto jurídicos, sociales y tributarios; que a su vez estando de acuerdo con Alberto Gago y Xavier Labandeira, mencionan que la OCDE considera a la tributación ambiental como instrumento para la modificación del comportamiento negativo que tengan los agentes contaminantes, por el mercado y el incentivo que puede representar.
Las aseveraciones de CEPAL en conjunto con OCDE, realizadas en el 2017, cercioran cómo es que Perú está actualmente y la gran necesidad que tiene de corregir a las actividades de consumo que general el daño ambiental, bien aclarado por el Dr. Pazos, que representa al consumismo y el ciclo económico sin considerar los posibles daños ambientales causados al realizar las actividades económicas. De esta manera se hace más real la situación proclamada por Bybee en 1991, 17 años antes de la creación del Ministerio del Ambiente en Perú, a la que llamó como "emergencia planetaria”.

Por consiguiente, tal como se mostró en la sección resultados, los problemas en general que causan mayor daño son prioritariamente los relacionados a la contaminación del agua y aire. Lo cuales ya han tenido consecuencias cuantificables en términos de PBI o Producto Bruto Interno medidos por el Banco Mundial, también llamados costos de la degradación ambiental que afectan ya sea en el corto o largo plazo a la salud de la población y del planeta. Cabe resaltar que una de las limitantes de la presente investigación fue la falta de datos macro de análisis actual en Perú, ya que el mencionado trabajo del Banco Mundial fue realizado en el 2007.

Surge, en consecuencia, la necesidad de realizar estudios relacionados al tema por cada uno de los factores ambientales más críticos. La agenda para futuras investigaciones debería tratar de responder interrogantes como: ¿Cuál es la relación que existe entre el bienestar social, el PBI y los costos de degradación ambiental? ¿Qué países usan actualmente la tributación ambiental como medio efectivo ante la contaminación? ¿Cómo influyen los instrumentos económicos en el com- 
portamiento de las personas? ¿En qué medida ha sido afectado el PBI anualmente en el periodo 2000-2017 por los costos de degradación ambiental?

En conclusión, es importante empezar a utilizar de manera efectiva la herramienta de la tributación o fiscalidad ambiental para, principalmente, atacar los comportamientos negativos generadores de contaminación que derivan en problemas ambientales que cada vez se hacen más evidentes e intensos en Perú y traen pérdidas económicas que afectan en mayor medida a las personas de escasos recursos; además para compensar el daño ambiental causado, de acuerdo al principio llamado contaminador-pagador. Adicionalmente, traerá como beneficios el aumento de la recaudación tributaria, acciones en favor del medioambiente, ingreso y desarrollo de tecnologías limpias al país, alineamiento con los ODS -Objetivos de Desarrollo Sostenible- de la ONU y una economía peruana que aplique medidas favorables para el desarrollo sostenible.

\section{REFERENCIAS BIBLIOGRÁFICAS}

Banco Mundial (2007). Análisis Ambiental del Perú: Retos para un desarrollo sostenible. Unidad de Desarrollo Sostenible Región de América Latina y el Caribe. Washington D.C., Editorial Banco Internacional de Reconstrucción y Fomento.

Bator (1988) Anatomy of Market Failure, The Quarterly Journal of Economics, cit. por: BAUMOL, W. y OATES, W "La teoría op. cit., p. 17. Citado por: JIMENEZ HERNANDEZ, Jorge. El tributo como instrumento de protección ambiental. Granada, Madrid : Editorial Comares.

Comisión Mundial El medio ambiente y desarrollo - CMMAD (1988). Our Common Future. Madrid: Alianza.

CEPAL/OCDE - Comisión Económica para América Latina y el Caribe / Organización de Cooperación y Desarrollo Económicos, Environmental Performance Reviews: Peru 2017. Paris: OEC Publishing. Recuperado de: https://read.oecd-ilibrary. org/environment/oecd-environmental-performance-reviews-peru_9789264283138-en\#page1

MEF elevó el Impuesto Selectivo al Consumo a los combustibles dié- sel. (11 de noviembre de 2016). RPP Noticias. Recuperado de https:// goo.gl/NQmjbq

Salud ambiental. (s. f.). Nota informativa en Temas de Salud de la OMS Organización Mundial de la Salud. Recuperado de http://www.who. int/topics/environmental_health/ es/

Pazos, C. (2004). La Globalización Económica Neoliberal y la Guerra: antagonistas esenciales del desarrollo sostenible y de la salud. La Habana: Centro Félix Varela.

Pimentel , G. \& Palacios , O. (22 de marzo de 2017). El agua es un bien escaso que el Perú no sabe administrar. El Comercio. Recuperado de https://goo.gl/iPfpPu

Prindle, R. (1966). Importancia del saneamiento ambiental para la salud de la comunidad. Washington, DC: Boletín de la Oficina Sanitaria Panamericana

Yacolca, D. (2012). Reforma Fiscal Verde para Sudamérica. Edición de la V Beca de Investigación CIATIEF-AEAT. Madrid: Instituto de Estudios Fiscales del Gobierno Español. Recuperado de http://webdms. ciat.org:8080/action.php?kt_path_ info=ktcore.actions.document view\&fDocumentId $=8183$ 